\title{
A GALOIS CONNECTION FOR REDUCED INCIDENCE ALGEBRAS
}

\begin{abstract}
ROBERT L. DAVIS
ABSTRACT. If $N=\{1, \cdots, n\}, D \subset N \times N$, and $F$ is an equivalence relation on the "entries" of $D$ the reduced incidence space $g(F)$ is the set of all real matrices $A$ with support in $D$ and such that $a_{i j}=a_{r s}$ whenever $(i, j) F(r, s)$. Let $\mathscr{L}(D)$ be the lattice of all subspaces of $R_{n}$ having support contained in $D$, and $\mathcal{E}(D)$ that of all equivalences on $D$. Then the map $g$ defined above is Galois connected with a map $f$ which sends a subspace $S$ into the equivalence $f(S)$ having $(i, j)[f(S)](r, s)$ whenever all $A$ in $S$ have $a_{i j}=a_{r s^{*}}$. The Galois closed subspaces (i.e. reduced incidence spaces) are shown to be just those subspaces which are closed under Hadamard multiplication, and if $S=g(F)$ is also a subalgebra then its support $D$ must be a transitive relation. Consequences include not only pinpointing the role of Hadamard multiplication in characterizing reduced incidence algebras, but methods for constructing interesting new types of algebras of matrices.
\end{abstract}

In Doubilet, Rota and Stanley's study [3] (hereafter referred to as D-R-S) of incidence algebras and how they lead to a coherent theory of generating functions the "main working tool" is the notion of reduced incidence algebra. One of the authors' theorems characterized such reduced incidence algebras, among certain subalgebras of the incidence algebra $I(P)$ of a partial order $P$, in terms of a pointwise multiplication which seems to have no other use in the theory.

When the partial order is finite the incidence algebra can be taken to be an algebra of matrices under ordinary matrix multiplication, while pointwise multiplication is usually called Hadamard multiplication. This note elucidates the somewhat surprising role of Hadamard multiplication by considering arbitrary subspaces of the vector space of all real $n \times n$ matrices. Those which are in a natural way "reduced incidence spaces" are just those which are Galois closed in a simple Galois correspondence. Transferring the D-R-S

Received by the editors July 17, 1972 and, in revised form, August 11, 1973. AMS (MOS) subject classifications (1970). Primary 06A 10; Secondary 06A15, $15 \mathrm{~A} 30$.

Key words and phrases. Reduced incidence algebra, incidence algebra, Galois connection, Hadamard product. 
methods to this more general situation permits deduction, in the finite case, of their theorem (under weaker hypotheses) and yields further information about new types of matrix algebras.

2. The basic type of Galois correspondence. Let $M_{n}$ be the space of all real $n \times n$ matrices. For $N=\{1,2, \cdots, n\}$ let $D \subseteq N \times N$ be any fixed subset of "entries" (an "incidence pattern", or relation on $N$ ). It is convenient to write " $i j$ " for the ordered pair (entry) $(i, j)$.

The support, Supp $A$, of any $A$ in $M_{n}$ is the set of entries $i j$ for which $a_{i j} \neq 0$, and if $S$ is any subspace of $M_{n}$ its support is the union Supp $S=$ U $\{$ Supp $A: A \in S\}$. For any $D$ in $N \times N$ it is natural to call the vector space, $I(D)$, of all $A$ with Supp $A \subseteq D$ the incidence space of the relation $D$.

For any given $D \subseteq N \times N$ the set $\mathscr{Q}(D)$ of all those subspaces $S$ of $M_{n}$ having Supp $S \subseteq D$ is closed under intersection and sum and hence is a sublattice of the lattice of all subspaces of $M_{n}$.

Call $\mathcal{E}(D)$ the lattice of all equivalence relations on the set $D$, ordered by relation inclusion (here denoted by $\leq) . \mathcal{E}(D)$ is a well-known complete lattice with infs given by intersection and sups as the intersections of all simultaneously containing equivalences.

Now for each $A$ in $I(D)$ write $E_{D}(A)$ (or just $E(A)$ if there is no need to emphasize the role of $D$ ) for the inverse-image equivalence defined on $D$ by $A: E_{D}(A)=\left\{(i j, r s) \in D \times D: a_{i j}=a_{r s}\right\}$. Then if $F$ is any equivalence in $\varepsilon(D)$ the set of " $F$-matrices" is $g_{D}(F)=g(F)=\left\{A \in I(D): E_{D}(A) \geq F\right\}$.

On the other hand if $S$ is any subspace in $\mathcal{L}(D)$ the "equivalence defined on $D$ by $S$ " is: $f_{D}(S)=f(S)=\bigcap\left\{E_{D}(A): A \in S\right\}$. Clearly $i j[f(S)] u v$ if and only if $a_{i j}=a_{u v}$ for every $A$ in $S$.

Proposition 1. For each subset $D$ of $N \times N$ the pair $\left(f_{D}, g_{D}\right)$ defines a Galois connection between $\mathcal{Q}(D)$ and $\mathcal{E}(D)$ in which the map $g$ is injective and $f$ surjective; furthermore, any $g(F)$ may be characterized as the largest subspace $S$ in $\mathcal{L}(D)$ for which $f(S)=F$.

Proof. Clearly each $f(S)$ is in $\mathcal{E}(D)$, being an intersection of equivalences on $D$. On the other hand, if $A$ and $B$ are in $I(D)$ with both $E(A)$ and $E(B) \geq F$ and if $c$ and $d$ are any real numbers then $c A+d B$ is in $I(D)$ and $E(c A+d B) \geq F$, so each $g(F)$ is a subspace in $\mathscr{L}(D)$.

It is easy to see that both $f$ and $g$ are anti-isotone (nonincreasing) functions, and also that the composites are extensive: $f g(F) \geq F$ and $g f(S) \geq$ $S$ for all $S$ and $F$.

In fact, for any $F$ in $\mathcal{E}(D)$ there is an $A$ in $I(D)$ with $E(A)=F$. (If $F$ 
has $k$ equivalence classes then such an $A$ can be written down using the integers $1,2, \cdots, k$ to mark the classes.) Thus it is clear that for any $F$, $f g(F)=\{E(A): E(A) \geq F\}=F$, so that $g$ is injective and $f$ surjective.

Hence (see, e.g. [1]) $f$ and $g$ define a Galois connection; $f g$ and $g f$ are closure operators on $\mathcal{E}(D)$ and $\mathcal{L}(D)$ respectively. The closure lattices $\mathcal{L}(D)$, consisting of spaces $S$ for which $g f(S)=S$, and $\overrightarrow{\mathcal{E}}(D)$ (which in this instance is all of $\varepsilon(D)$ ) are anti-isomorphic under the inverse maps $\bar{f}$ (restriction of $f$ to $\overline{\mathcal{Q}}$ ) and $\bar{g}=g$.

The last statement of the proposition is now immediate.

Using this framework depends on identifying the "Galois closed" subspaces of $\mathfrak{L}(D)$. To this end denote by $A * B$ the Hadamard product of matrices $A$ and $B$ (that matrix whose $i j$ entry is $a_{i j} b_{i j}$ ) and call a set of matrices "Hadamard closed" if it is closed under this product.

Theorem 2. A subspace $S$ in $\mathscr{L}(D)$ is Galois closed if and only if it is Hadamard closed.

Proof. To say $S$ is Galois closed is to say $S$ is precisely the set of all $F$-matrices, where $F$ is the equivalence defined by $S$. Then if $A, B$ are in $S$ it is clear that $A * B$ is an $F$-matrix, therefore in $S$.

For the converse, let $f(S)=F$ have equivalence classes $K, H, \cdots$, in $D$. It suffices to show that $S$ contains each "indicator" $E_{K}=\Sigma\left\{E_{i j}: i j \in K\right\}$, since these are a basis for $g(F)$. This can be seen by essentially the same proof as given by D-R-S for the more restricted case treated in their theorem (Corollary 5 below).

Just as in their proof it can be shown that for any distinct classes $H$ and $K$ there is a matrix $C=C_{H, K}$ in $S$ which is nonzero on entries in $K$ and zero on entries in $H$. Hence the Hadamard product of all such $C_{H, K}$ with $H \neq K$ is (apart from a scalar multiple) the indicator $E_{K}$, and it lies in $S$ by Hadamard closure.

3. Multiplicativity. In many applications the interesting subspaces of $M_{n}$ are those which are subalgebras. The questions whether a subspace $S$ is closed under Hadamard multiplication, and whether $S$ is closed under matrix multiplication, are essentially independent. There are subspaces $S$ that are both, others that are neither, and others enjoying either one of these types of closure without the other.

Proposition 3. If the subspace $S$ of $M_{n}$ is Hadamard closed and also closed under matrix multiplication then $D=\operatorname{Supp} S$ must be a transitive relation. 
Proof. The key here is that Hadamard closure of any subset $S$ guarantees that for any $A$ in $S$ with a given support there is a nonnegative matrix $A^{\prime}$ in $S$ with the same support, to wit, $A^{\prime}=A * A$. Then if $i j$ and $j k$ are any elements of Supp $S$ there are nonnegative matrices $A$ and $B$ with $a_{i j}>0$ and $b_{j k}>0$, so $A B=C$ has $c_{i k}=\sum a_{i j} b_{j k}>0$, hence $i k \in \operatorname{Supp} S$.

(It is easy to construct subalgebras of $M_{n}$ with nontransitive support. One example is the algebra $S$ generated by $E_{12}+E_{13}=A$ and $B=E_{24}-$ $E_{34^{\circ}}$ )

While for each $F$ in any $\mathscr{E}(D)$ the set $g(F)$ of all $F$-matrices is always an Hadamard closed vector space, it is quite unlikely to be a subalgebra even if $D$ is transitive. Any $F$ for which $g(F)$ is closed under multiplication will be called a multiplicative equivalence.

Proposition 4. If $D$ is a transitive relation the set $\mathscr{G}_{m}(D)$ of multiplicative equivalences on $D$ is itself a lattice, with sups defined as in $E(D)$. (Since infs are not in general the same it is not a sublattice of $\mathcal{E}(D)$.)

Proof. This proposition, which for the special case where $D$ is a partial order was given a longer but direct proof by D-R-S, is in fact an immediate consequence of the anti-isomorphism of $\xi(D)$ and $\bar{\complement}(D)$. If $F$ and $G$ are any two multiplicative equivalences then $S=g(F)$ and $T=g(G)$ are Hadamard closed subalgebras of $M_{n}$. Thus their intersection $S \cap T=g(F) \cap g(G)=$ $g(F \vee G)$ is a subalgebra and so $F \vee G$ is, by definition, multiplicative. Since $D$ is transitive the minimal equivalence on $D$, whose $g$-image is just $I(D)$, is also multiplicative as was shown in [2]. Hence $\mathscr{E}_{m}(D)$ is dual to a "closure system" or "Moore family" (see [1, p. 111]) and therewith a complete lattice with sup, $F \vee G$, the same as in the lattice of all equivalences. The inf, $F \wedge G=\bigvee\left\{H \in \mathcal{E}_{m}(D): H \leq F, H \leq G\right\}$, need not be the same as that in the lattice of all equivalences.

4. The D-R-S theorem. The incidence space $I(D)$ of any relation $D$ is the image $g_{D}\left(F_{0}\right)$ of the minimal equivalence $F_{0}$ on $D$. It is natural to describe as a reduced incidence space on a relation $D$ the image $g_{D}(F)$ of any equivalence $F$ on $D$. Theorem 2 shows that the subspaces of $M_{n}$ which are reduced incidence spaces are just those which are Hadamard closed, while Proposition 3 shows that if $S=g_{D}(F)$ is also a subalgebra then $D$ must be a transitive relation and $F$ a multiplicative equivalence on $D$. In other words a reduced incidence algebra is in general any image $g_{D}(F)$ of a multiplicative equivalence on any transitive relation $D$. This leads to an alternative statement of Proposition 3. 
Proposition 3'. A subalgebra $S$ of $M_{n}$ is Hadamard closed if and only if $S$ is the reduced incidence algebra $g_{D}(F)$ of some multiplicative equiva. lence on a transitive relation $D=$ Supp $S$.

The original D-R-S theorem concerns a given partial order $P$ on $N$ and subalgebras of its incidence algebra that contain the indicator or zeta matrix, $Z$, of $P\left(z_{i j}=1\right.$ if $i j \in P$ and $z_{i j}=0$ if not $)$.

Corollary 5 (Doubilet-Rota-Stanley). A subalgebra $S$ of $I(P)$ containing the zeta matrix $Z$ for $P$ is the reduced incidence algebra $g_{P}(F)$ for some multiplicative equivalence $F$ on $P$ if and only if $S$ is Hadamard closed.

(D-R-S assume in addition that $S$ contains the identity matrix $I$, but this is unnecessary. Any Hadamard closed subalgebra containing $Z$ must also contain $I$ as can be seen by considering differences of scalar multiples of various Hadamard powers of $Z$.)

It is clear that if $F$ is a multiplicative equivalence on the partial order $P$ then the Hadamard closed space $g_{P}(F)$ contains $Z$. If, on the other hand, $S$ is a subalgebra of $I(P)$ containing $Z$ and is also Hadamard closed then Proposition $3^{\prime}$ shows $S=g_{D}(F)$ for a multiplicative equivalence $F$ on some transitive relation $D=\operatorname{Supp} S$. But since $Z \in S, P \leq D$; and since $S$ is contained in $I(P), D \leq P$.

For the many applications based on Möbius functions an incidence algebra $S$ must have its support $D$ not merely transitive but a partial order on $N$ so that its zeta function is invertible. To guarantee that $D$ is some partial order it would suffice to assume $S$ contains the identity and, as above, that $S$ is contained in the incidence algebra of some (other) partial order, or again, just that $D \cap D^{\prime}=I$, where $D^{\prime}$ is the transpose. An alternative approach is simply to put reasonable hypotheses on the zeta function itself.

Proposition 6. Let $S$ be a Hadamard closed subalgebra of $M_{n}$ containing the zeta matrix $Z$ of its support $D=\operatorname{Supp} S$. Then $S$ is the reduced incidence algebra of a partial order $P(=D)$ on all of $N$ if and only if the rank of $Z$ is $n$.

Since det $Z \neq 0$ there is a permutation relation $Q$ sending $i \rightarrow j_{i}$ such that $z_{1 j_{1}} z_{2 j_{2}} \cdots z_{n j_{n}} \neq 0$. Then $Q^{k} \leq D^{k}$ for each $k$; but then $Q^{n}=I \leq D^{n}$, whereas, since $D$ is transitive, $D^{n} \leq D$, which therefore is reflexive. (These are all relation inequalities.) Now if the equivalence $D \cap D^{\prime}$ were not the identity then two or more rows of $Z$ would be the same, so its rank could not be $n$. The converse is immediate. 


\section{BIBLIOGRAPHY}

1. Garrett Birkhoff, Lattice theory, 3rd ed., Amer. Math. Soc. Colloq. Publ., vol. 25, Amer. Math. Soc., Providence, R. I., 1967. MR 37 \#2638.

2. R. L. Davis, Algebras defined by patterns of zeros, J. Combinatorial Theory 9 (1970), 257-260. MR 42 \#3107.

3. Peter Doubilet, G.-C. Rota and R. P. Stanley, On the foundations of combinatorial theory. VI. The idea of generating function, Proc. Sixth Berkeley Sympos. Math. Statist. and Probability, vol. 2, Univ. of California, Los Angeles, Calif., 1972, pp. 267-318.

4. D. A. Smith, Incidence functions as generalized arithmetical functions. II, Duke Math. J. 36 (1969), 15-30. MR 39 \#084.

DEPARTMENT OF MATHEMATICS, UNIVERSITY OF NORTH CAROLINA, CHAPEL HILL, NORTH CAROLINA 27514 